\title{
La elegía y lo elegíaco en La Dorotea. Poética y desconsuelo
}

La elegía y lo elegíaco en La Dorotea. Poética y desconsuelo

Florencia Calvo

Instituto de Filología y Literaturas Hispánicas "Dr. Amado Alonso" Universidad de Buenos Aires- CONICET, Argentina

\section{Resumen:}

Este artículo complementa una serie de trabajos anteriores en los que intento problematizar algunas cuestiones relacionadas con el género de La Dorotea en cruce con su momento de producción. Entendiendo ya agotadas de alguna manera las posibilidades de reflexionar acerca de la especificidad del sintagma "acción en prosa" postulo en estos acercamientos previos que la obra funciona como una geografía textual de la desintegración o de la ruptura. Desde este ángulo considero que se pueden sumar elementos para la definición de esta complicada ultima época del poeta, su extensamente analizada senectute, en tanto La Dorotea tendría mucho para decir no tanto tal vez en relación con su experiencia vital sino en relación con posicionamientos poéticos previos, con experimentaciones teóricas o como una forma de relativizar lo ya escrito antes de dejar de escribir. En este caso me interesa detenerme particularmente en lo que propongo llamar la poética del desconsuelo como territorio para explorar el manejo que el texto hace de los vectores propios del género elegíaco, con el objeto de ver si es posible establecer desde allí algunas conclusiones que colaboren en la intelección del universo lopiano.

Palabras Clave: Lope de Vega, La Dorotea, Elegía, Parodia.

\section{ABstract:}

The aim of this paper is to present a reading of La Dorotea, one of the latest books of Lope de Vega, leaving apart the biographical approachs and the idea of the text only as a continuation of Celestina. Thus, we intend to analyze in these pages another way, reading a poetry of despair and the presence of the elegiac gender in order to establish some conclusions that contribute to the intellection of Lope de Vega 's universe.

KeYworDs: Lope de Vega, La Dorotea, Elegiac gender, Parody.

\section{I}

En este trabajo me propongo deslindar algunas cuestiones relacionadas con el género de La Dorotea, una de las obras más complejas de Lope de Vega, en cruce con su momento de producción, es decir la última etapa de su autor. Agotadas ya las posibilidades de reflexionar acerca de la especificidad del sintagma "acción en prosa", de su identificación con el modelo celestinesco o de su complejidad argumental, entiendo que la obra funciona como una geografía textual de la desintegración o de la ruptura. ${ }^{1}$ Desde este ángulo considero que se pueden sumar elementos para la definición de esta complicada época del poeta, su extensamente analizada senectute en tanto La Dorotea tendría mucho para decir sobre los últimos años de Lope; no tanto tal vez en relación con su experiencia vital sino en relación con posicionamientos poéticos previos, con ciertas polémicas literarias o con experimentaciones teóricas. Además de todas esas variables, comunes en otras obras de Lope, mi recorrido por La Dorotea propone entenderla también como una suerte de manifiesto en el que su autor relativiza su propia obra anterior, lo ya escrito, antes de dejar de escribir. Del mismo modo que con el heterónimo Tomé de Burguillos, Lope recorre su trayectoria poética, así en La Dorotea traza un derrotero por la totalidad de su producción como poeta, como narrador y como dramaturgo. Concluía al respecto en dichos trabajos que tanto la comedia lopesca, sobre todo la urbana, como las Novelas a Marcia Leonarda se resignifican en el texto desde una mirada irónica y desintegradora de una serie de tópicos narrativos y de núcleos argumentales que transforman al texto en una nada vertiginosa.

Pero es evidente que La Dorotea además de funcionar como un espacio en el que afloran textos anteriores del propio Lope también echa mano de diferentes convenciones genéricas para ponerlas en cuestión o por 
lo menos para reflexionar de alguna manera sobre ellas. En general la literatura barroca va reformulando de manera problemática no solo las convenciones de los géneros heredados tanto del Renacimiento como de la Antigüedad clásica, sino también sus límites; y en el caso de Lope en particular este gesto puede verse en sus producciones de senectute, sobre todo en sus últimos poemas en los que no quedan del todo especificadas las fronteras formales tal como puede verse, por ejemplo, en los estudios introductorios a sus Elegías y Elogios editados por Felipe Pedraza (2010). En este caso me interesa detenerme particularmente en lo que propongo llamar la poética del desconsuelo como territorio para explorar el manejo que el texto hace de los vectores propios del género elegíaco, por ser tal vez este género el más claro para ver la laxitud de los límites formales.

La elección no es azarosa; antes de entrar de lleno en el problema debemos desbrozar de algún modo qué es lo que se podría entender por lo elegíaco y su relación con la elegía. Es sin duda en los acercamientos hacia el género realizados por el grupo PASO donde encontramos los mejores elementos para esta descripción. Así Begoña López Bueno indica, en su introducción al volumen en el que se agrupan los trabajos que analizarán la elegía desde la Antigüedad clásica hasta el siglo XVII español, que la elegía "no se trata de un género perfectamente definido en sus límites y la contaminación es su rasgo constitutivo más considerable”. (1996:7)

Del mismo modo para la estudiosa:

[...] la elegía es sobre todo un modo poético atenido a la forma (a la forma métrica del dístico elegíaco), en las literaturas contemporáneas la elegía se asocia más a una sustancia (o, si se quiere, a una forma sustantiva): es la poesía de la pérdida y lamentación consiguiente. Aunque por debajo de ambas polaridades o extremos hay un terreno compartido que vertebra y da unidad y sentido a toda la historia occidental de la elegía: las nociones tonales de ausencia, melancolía y nostalgia, el ámbito -si así quiere llamarse- de la sentimentalidad. Lo que requiere, a su vez, de propio y por esencia, el espacio de lo privado, de la intimidad. (Aspectos todos ellos-y valgan como un primer acercamiento al género- que se oponen respectivamente a lo celebrativo y a lo público, las dos nociones precisamente asociadas al género de la oda -aunque la oda española áurea más genuina no se atenga precisamente a ello-, que así actúan, oda y elegía, como una pareja antitética y complementaria, esto es, dialéctica, en la tradición poética occidental.) (1996:8)

No es muy difícil, entonces, vistas estas categorías constitutivas de lo elegíaco y la gran cantidad de acercamientos que definen las características sobre la última época del poeta pensar que es un eje plausible de vertebrar la senectute lopesca. En el mismo encuentro sobre la elegía, en un artículo también publicado en dicho volumen, Yolanda Novo, realiza una suerte de historia del género. Se detiene luego a pensar los modos de la elegía específicamente en Lope para definir que "cristaliza en el autor en el último tercio del XVI y que Lope intensifica esa naturaleza intergenérica inicialmente atenuada por Garcilaso, el primer dechado vernáculo para nuestro Siglo de Oro, y, más tarde, entre otros, por y luego por Herrera" (1996:231-232). En ese contexto Novo afirma que "se instaura un tipo de elegía permeable en cantidad y calidad a otras órbitas genéricas que en la mayoría de los casos es dificilmente, ya reconocible como un género poético y cuya morfología delata una poética susceptible de irradiar y de dejarse contaminar por otros géneros" (1996:232-233). Luego afirma, en consonancia con Juan Manuel Rozas (1990) en su ya citado fundacional artículo sobre la senectute de Lope, que en el período comprendido entre 1627 y 1635 caracterizado -como todos sabemos- por el ascetismo ético, el desengaño, la moral neoestoica, los ecos horacianos y virgilianos, se verifica además la funcionalidad de todos estos ejes en relación con la elegía para concluir que:

Sin duda el ascetismo ético afecto al desengaño, la moral estoica y el aurea mediocritas de cuño virgiliano y horaciano son comunes a todas las composiciones de ahora en tanto sustratos literarios cimentadores de una nueva poética: la de la autoironía y deconstrucción paródica de fórmulas, moldes y géneros desgastados, sobre todo los propios, y la de la retórica de la máscara poética llevada a su apogeo bajo la faz, ahora, del poeta grave, filosófico, relegado y escéptico. No extraña, pues, que muy en particular las fechas últimas del Lope anciano (1631-1635) se correspondan con la conformación más sincrética y arriesgada de la elegía, cuya producción, además, se incrementa, casi siempre al abrigo del asunto funeral. (Novo, 1996:248) 
Yolanda Novo va a analizar más adelante cada una de las obras que incluye bajo este rótulo. De La Dorotea selecciona, del mismo modo que Juan Manuel Rozas, las transitadas barquillas, una serie de idilios piscatorios incluidos en el acto tercero de la obra.

Acerca de las barquillas Novo opina que convendría revisar su intertextualidad con la égloga a Amarilis y con el soneto "en seso" de Burguillos, aquel que comienza "Resuelta en polvo...", ambos de la misma época. Concluye que en ellas la materia fúnebre está formalizada novedosamente en conjunción con los estereotipos de la "soledad" poética barroca sumado al artificio autobiográfico del yo filosófico. (251)

También Rozas (1990:107) había propuesto catalogar como elegías a las cuatro barquillas insertas en la obra, puestas siempre en boca del personaje de Julio, quien las refiere hechas por "un gentilhombre que tú conoces, se le ha muerto su dama" en el que la crítica ha visto por supuesto al propio Lope. Del mismo modo que para Yolanda Novo las barquillas tienen como punto de origen la muerte de Marta de Nevares pero la trascienden inmediatamente y postulan otros temas más generales e involucrados con la elegía más allá de la materia fúnebre. De esta manera Rozas señala que:

El 7 de abril de 1632 Lope sufre el mayor de los reveses del ciclo de vejez. Muere su último gran amor [...] Como sabemos, el poeta se puso a escribir inmediatamente una serie de elegías que constituyen algunos de sus mejores poemas de todos los tiempos y que incluirá en La Dorotea [...] escribe en pocos días esa serie de barquillas en las que el tema central no son los amores ni la figura de Marta sino la soledad en que el poeta queda sin ella. Son elegías de autoformación y autoconsuelo escritas con tanta elevación de espíritu como de forma. (1990:107)

Antonio Sánchez Jiménez (2006) va un paso más allá en la relación entre barquillas y materia fúnebre y demuestra que las barquillas no serían piezas escritas en esa circunstancia sino que la muerte de Marta desplaza la intencionalidad primera de los poemas. Así:

los romances de 1632 abandonan la imagen de un poeta preocupado por la recepción de su obra en la tormenta de los criticos, imagen tópica entre los poetas latinos (Curtius, 128-29). Frente a este significado puramente literario, las barquillas de $L a$ Dorotea apuntan a una temática amorosa y vital típica del ciclo de senectute de Lope. Como en El huerto deshecho en las barquillas las inclemencias del tiempo representan las desgracias. (2006:338).

Ya sea escritas para la ocasión de la muerte de Marta que les daría por el hecho en sí mismo su carácter élego, ya sea resignificadas por el desplazamiento temático (del poeta incomprendido al amante que llora la muerte de su amada) o por los contextos en que se insertan (recorridos por Ximena González en este mismo volumen) es evidente que entre los romancillos al modo piscatorio y el texto en prosa de La Dorotea, sumado a que la intermediación entre ambos es el personaje de Julio, en su perfil serio, se produce una relación de ida y vuelta en la construcción del discurso elegíaco. Así, en las barquillas la elegía se resignifica a la luz de su interacción con el multifacético espesor del texto de la obra y contribuye a la problematización general acerca de su especificidad.

Para entender esta capacidad o esta complejización de la elegía se puede cerrar este recorrido teórico con las consideraciones de Pedro Ruiz quien, entre otras consideraciones, indica que:

En el barroco se da, naturalmente, la persistencia clasicista y retórica de la elegía funeral en tercetos pero se impone la búsqueda de la expresión de lo funerario y o elegíaco por otros medios. La elegía que deriva cada vez más hacia lo elegíaco entendido como un tono que rebasa con particular intensidad lo que sería una estricta codificación genérica. (1996: 323)

II

Todas estas afirmaciones y especulaciones teóricas no hacen más que confirmar las directrices de la senectute lopesca y la pertinencia de adjudicarles a las barquillas el carácter de elegías junto con el resto de las elegías de la última época de Lope. ${ }^{2}$ Sin respetar una forma estrófica o una estructura retórica La Dorotea da cabida así a un nuevo género que se funde en la diversidad que determina la poética del texto. 
Las barquillas, lugar de la elegía en La Dorotea para los críticos que han intentado cierta codificación de dicho género en Lope, además de constituir el mejor vehículo para la expresión del dolor y de toda esa serie de sentimientos propios de lo eglógico, colaboran en la multiplicidad genérica de un texto que escapa a los rótulos tradicionales. Por otra parte las barquillas parecerían, desde su categoría de lamento fúnebre, alzarse con la exclusividad de la representación autobiográfica en tanto remitirían al episodio biográfico nodal y arrasador no solo en la vida del poeta sino también en la estructura del relato: aquello que el texto no nombra, la muerte de Marta de Nevares.

Pero más allá de esto, no debemos perder de vista que las barquillas funcionan dentro de un nivel particular de la obra y de los niveles de representación, de sus estructuras argumentales, temporales y espaciales de la historia; en la que diferentes núcleos textuales dilatan, impiden o directamente clausuran las posibilidades de una organización lineal y coherente.

$\mathrm{Y}$ es en este punto me parece interesante pensar las distintas caras que adquiere lo elegíaco en el texto más allá de esta clara circunscripción puntual a las cuatro barquillas. La hipótesis es que, ahondando en este funcionamiento, se podría establecer alguna línea más que permita desentrañar ciertos rasgos constructivos no solamente de la obra sino también de este último momento de producción de su autor.

\section{III}

Hay una serie de parlamentos de Dorotea en los que también es factible encontrar cierta tonalidad elegíaca si acordamos con los modos que dicha tonalidad puede adquirir.3 Uno de ellos puede verificarse en el siguiente ejemplo:

El oro la ha vencido, Gerarda lo ha tratado, entre las dos se consultó mi muerte.;Oh cruel sentencia! Supo que habia vendido los pasamanos del manteo de tela el mes pasado y antiyer el de primavera de flores. Dice que es para darte el dinero que juegues, como si tú jugases, siendo tu mayor vicio libros de tantas lenguas; y que con versos me engañas, y con tu voz, como sirena me llevas dulcemente al mar de la vejez, donde los desengaños sirvan de túmulo y el arrepentimiento de castigo. iAy Dios! ¡Ay de mí! Déjame deshacer estos ojos pues ya no son tuyos; no hay que respetarlos, no me ha de gozar con ellos quien ella piensa, porque verá en sus niñas tu retrato que sabrá defenderlos. ¡Ay Dios! ¡Ay muerte! (48). ${ }^{4}$

Vemos cómo en Dorotea el lamento es siempre un lamento de amor. Así se despliega la línea de lo elegíaco en el personaje y se caracteriza por la pérdida amorosa en un principio y se refuerza luego a lo largo del texto por esta suerte de triángulo amoroso que nunca termina de desplegar toda su potencialidad argumental y que solo parecería estar regido por los lamentos de sus integrantes: Dorotea, Marfisa o Fernando se incluyen en una cadena de pérdidas amorosas que en la mayoría de sus casos están ligadas por las lágrimas.

Dorotea es quien protagoniza la mayoría de esos parlamentos de lamentaciones y muchas veces estos se hacen explícitos en la contaminación y en la mezcla de géneros variados como, por ejemplo, el género epistolar. Dorotea representa como personaje, de alguna manera en otro nivel del texto, el lamento de las barquillas.

Marta Cuenca-Godbert (2010) analiza el lenguaje de las lágrimas en la obra y lo considera formado por significaciones varias; reivindica la funcionalidad de las lágrimas fúnebres, que desprende de las barquillas pero que adjudica también a los personajes. Las lágrimas cohesionarían de algún modo un texto que se define a sí mismo como desordenado. ${ }^{5}$

Si seguimos a Pedro Ruiz Pérez las lágrimas también son la cifra de la elegía, así se expresa en el artículo ya citado confirmando esta relación entre lo elegíaco y las lágrimas al señalar que:

La elegía comienza a dar cabida a lo confesional, lo cotidiano y lo anecdótico: la ampliación de alma, la sentimentalidad burguesa un nuevo sentido de la historia, la vida y la muerte y el propio concepto del hombre, su expresión poética se desplaza entre las lágrimas. (1996:324) 
Es decir que Dorotea y las barquillas quedan unidas por las lágrimas y por el tono elegíaco de ambos lamentos. En el triángulo en el que discurre su historia amorosa Marfisa también participa de estos lamentos por el amor perdido. Y Fernando, personaje a veces identificado con Lope, voz masculina más autorizada para reflexionar en términos metaliterarios (como ya ha acostumbrado Lope a sus lectores en las Novelas a Marcia Leonarda), es quien hace explícitos los códigos genéricos de la elegía no solo al funcionar como poeta de las barquillas sino también al escribir bajo la forma de soneto un epitafio a la tumba del retrato de Dorotea (Acto IV, escena primera) que acaba de enterrar.

Si bien el enterrar el retrato es un tópico literario creo que es fundamental este soneto a la tumba de un retrato en el mismo acto en el que más adelante se reflexionará sobre la poesía. Una lectura en clave metapoética del soneto la lleva adelante Marta Cuenca-Godbert en el artículo ya citado. Allí la estudiosa concluye que:

Les larmes sont une humeur qui console, mais elles sont également une encre indélébile, un instrument de mythification. Dans La Dorotea, l'expression de la douleur est à la fois manifeste et en sourdine.L'encre indélébile des larmes, présentes partout de façon diffuse, fait de l'oeuvre toute entière un planctus retenu, contenu, écrit à la gloire de l'être aimé et à la gloire de soi. (2010:163)

Entonces, si las barquillas abren la elegía hacia la obra de Lope de senectute, más allá de La Dorotea, hacia adentro del texto la elegía se condensa en un soneto fúnebre escrito por Fernando a un retrato. Mientras tanto las lágrimas funcionan como el signo más claro de este espíritu élego que cruza el texto. Hasta aquí queda claro que hay una reflexión, ya sea desde el género, ya sea desde la construcción del lamento por la historia amorosa que coloca a la elegía y a todo lo que trae consigo retórica y poéticamente en un lugar privilegiado.Queda claro también que este gesto autoral es propio de La Dorotea pero también se hace extensivo al resto de los textos de este último período de Lope.

\section{IV}

Esta presentación de los códigos genéricos de lo elegíaco y su reflexión acerca de ellos puede verificarse en las barquillas, en las lágrimas o en los monólogos y los diálogos en los que los personajes se lamentan de sus desgracias amorosas. Todas estas variables muestran las posibilidades de la elegía más allá del llanto fúnebre.

Pero también puede verse aquí el otro gran gesto de este último Lope que es la parodia o la autoparodia. Hay, entonces, además de una diferencia de niveles de enunciación entre las barquillas y el modo del manejo de lo elegíaco entre los personajes, un giro hacia la parodia que no puede verse en los romancillos intercalados ya que allí se respetan los postulados genéricos. Sin embargo, en el giro hacia la trama argumental y su intermediación por parte de los personajes la elegía puede derivar hacia la parodia. Veamos algunos ejemplos. La primera cita de la obra que presentamos en estas páginas: aquella de Dorotea quejándose en la que quedaba claro que el lamento fúnebre se desplazaba hacia el lamento amoroso, siguiendo la evolución del paradigma de lo élego tal como lo apunta la crítica, corresponde a la escena quinta del Acto Primero en la que Dorotea va al encuentro de Fernando. La primera intervención de Dorotea en dicho intercambio se produce mediante las exclamaciones “iAy Dios! ¡Ay Muerte!” (45) y dichas expresiones producen lo que sería el primer diálogo entre los desdichados amantes:

DOROTEA: ¡Ay Dios! ¡Ay Muerte!

FERNANDO: Ya volvió a concertarse cuanto habías dejado descompuesto; ya el amor mata, ya el sol alumbra, ya la primavera se esmalta, y yo estoy vivo. Pero ¿cómo la primera palabra ha sido las dos cosas más poderosas, Dios y la muerte?

Mientras que Fernando construye su diálogo siguiendo la tópica de un diálogo amoroso, Dorotea que ya ha decidido que sus amores van a finalizar desastradamente, guía su discurso por los carriles de la lamentación. El largo parlamento, citado más arriba, finaliza como ha empezado su diálogo con Fernando. Y si bien ambos, tanto el discurso amoroso desplegado por Fernando como la queja de Dorotea, se construyen para reforzar 
una línea argumental que roza la tragedia, llevan los dos el germen de la autoparodia. En el caso de Dorotea es Julio quien en esta escena adquiere el papel del criado gracioso de la comedia, ${ }^{6}$ el que lo va a dejar claro ya que luego del segundo “ ¡Ay Dios, ay muerte!” de la dama y antes de que Fernando pueda continuar con su consuelo, expresa en un aparte "(Volvió al estribo)" (48). ${ }^{7}$

La intervención de Julio permite en primer lugar su identificación con un agente portador de comicidad en una pieza dramática en tanto está registrada como un aparte. Tiene una doble funcionalidad. Por un lado romper esa suerte de riesgo trágico que el diálogo entre los amantes vendría gestando y por otro desmontar la retórica del lamento dejando en claro cuáles son sus mecanismos constructivos. Ambas funciones dejan al descubierto los procedimientos constructivos de la materia elegíaca y por qué no, su ineficacia en esta obra.

Dijimos que otro lugar para verificar el distanciamiento de los procedimientos de la elegía es la escena en la que Fernando recita el soneto que ha escrito a la tumba del retrato de Dorotea. Del mismo modo que Sánchez Jiménez con las barquillas, Cuenca- Godbert lee este soneto en clave metaliteraria. Me interesa detenerme en un detalle que sirve a los efectos de mi argumentación a favor de leer la parodia en los códigos de la elegía. El episodio del soneto se introduce en la escena primera del Acto Cuarto. Allí Fernando relata su trágica historia de amor con Dorotea:

FERNANDO: No lo había menester mi corazón, porque sólo en él se halló con verdad el movimiento perpetuo. Finalmente determiné de quitarme la ocasión de tantas penas, porque ya no me servía de consuelo, sino de desesperación y sacando la daga...

FELIPA: ¡Jesús! ¿Matastes a Dorotea?

FERNANDO: Cavé la poca tierra que en el espacio de dos peñas estaba ociosa, y enterré el retrato, habiendo hecho primero estos versos. (272)

Felipa introduce la daga como objeto de muerte pero Fernando inmediatamente relativiza esta funcionalidad. Así, todo lo que rodea la muerte cambia su significado y deriva en el pretexto para un ejercicio de escritura. Ejercicio que para Cuenca-Godbert tiene que ver con la capacidad de la poesía de transformar su objeto en inmortal al renacer de sus cenizas. ${ }^{8}$ Para este recorrido que intento trazar aquí se acerca más a desmontar los modos de construcción de la materia elegíaca y su contaminación con el lamento amoroso. No sólo la daga sino la acumulación de imágenes de objetos de representación de lo funerario presentes en los cuartetos así lo demostrarían:

Aqui donde jamás tu rostro hermoso
planta mortal, divina Dorotea,
toque atrevida, tu sepulcro sea,
sin colunas de pórfido lustroso.
El fénix yace en inmortal reposo;
no vuelva a renacer, ni el sol le vea,
construyéndole en vez de urna sabea,
mis lágrimas pirámide oloroso. (272)

Pero tal vez donde mejor pueda verse esa parodia de lo elegíaco sea en el personaje de Gerarda y en su celestinesco llanto en la escena VI del Acto II. En Gerarda, definida en algunos rasgos en forma cercana al personaje de Celestina, las lágrimas van a estar siempre más cerca del vino que del agua:

GERARDA: Pues dame tú de beber, doncella de la Vera, y perdona, que ya sé que te traigo hecha pedazos.

CELIA: No quiere señora.

GERARDA: Este es tu hijo, don Lope, ni es miel ni es hiel, ni vinagre ni arrope

CELIA: En los ojos tienes eso postrero, como has llorado. (151)

y la única agua que existe es aquella que sirve para sorprender a los amantes en su adulterio: 
TEODORA: Gerarda, no bebas más, que dices desatinos. Y en otra parte pensarán que era verdad lo que dices. ¿Para qué lloras?

GERARDA: Porque fue crueldad llevarle a galeras.

CELIA: Ya lo enmienda.

GERARDA: Dios manda que se digan las verdades.

TEODORA: No es daño del prójimo.

GERARDA: ¿Qué daño es contar sus alabanzas, Teodora, ni refrescar la memoria del bien que se ha perdido?

CELIA: A lo menos refrescar lo bien que se ha bebido.

GERARDA: La primera vez que me halló en aquella niñería del estudiante, fue notable su paciencia. Era invierno y echónos a mí y a él un jarro de agua en la cama diciendo con aquella bondad de que él se preciaba mucho "A los bellacos, mojallos".

TEODORA: ¿no adviertes Dorotea la condición del vino? (147-148)

La oposición agua-vino es también la que la va a llevar a la muerte en las últimas escenas del Acto $\mathrm{V}$ en las que las acumulaciones de muertes y de lamentos logran también un claro efecto paródico. Me refiero a las escenas XI y a la última del Acto Quinto en donde todos los personajes entran, por un lado, en un espiral de muerte que conspira contra la verosimilitud pero lo hacen al mismo tiempo que se burlan de la muerte de Gerarda por buscar agua y no vino, bebida que ha caracterizado al personaje, a sus parlamentos y a sus acciones a lo largo de toda la obra.

TEODORA: ¿Qué voces son aquéllas, Felipa, y qué ruido? ¿Quién ha caído en la cueva?

FELIPA: ¡Ay, señora, en la voz es mi madre, que iba por agua para Dorotea, que se ha desmayado.

TEODORA: ¿No había de dónde más cerca pudiera traerla? ¡Qué buena diligencia para un desmayo!

CELIA: [...] ¡Oh miserable espectáculo! Gerarda es muerta. Mas ¿quién dijera que buscando agua? (399-400)

Gerarda, quien ha hecho de lo elegíaco una parodia transformando, entre otras cosas, las lágrimas en vino, pronuncia antes de morir lo que van a ser sus últimas palabras en la obra reflexionando sobre la elegía, sobre cómo decir la muerte.

GERARDA: ¡Ay, Laurencio! Bien pudieras escusar tan encarecido estilo de contar una desgracia, que bastaban las palabras sin las lágrimas, y los sentimientos sin los sollozos. Tenla esa mano, que le ha dado mal de corazón. Tenla, tenla, que se hará pedazos, mientras voy por agua. (399)

Para concluir vuelvo a Yolanda Novo, quien señala que:

Otra de las máscaras falsificadoras de autobiografismo predilecta en este período lopiano resulta irreconciliable con el modo elegíaco: la del poeta que se burla de toda la literatura seria de su tiempo a través de la parodia. Porque la elegía en su metamorfosis no podía llegar a ser burlesca. (1996:259)

Sin embargo, en una nota al pie expande esta afirmación e indica que:

Lo que no comporta que las directrices de lo elegíaco no puedan ser puestas en solfa, pero en otros moldes o géneros distantes de la elegía canónica, como el soneto. Así hace Lope, por ejemplo, en el soneto de su Burguillos dedicado a la muerte de una perra de agua ("Ala muerte de Timosca, perra de agua famosa; matóla la rueda de un molino"), o en aquel otro del mismo libro ("Propone lo que ha de cantar en fe de los méritos del sujeto") en que parodia a los elegíacos latinos al aludir a los nombres poéticos prototípicos de las amadas en sus poemas: la Corina ovidiana, la Cintia de Propercio. Llama la atención que la burla más abierta opere sobre lo elegíaco amoroso. Pues la del asunto fúnebre la restringe Lope al mundo animal -asimismo evidente en el soneto-epitafio a la sepultura de Marramaquiz, también en el Burguillos-, y en modo alguno la efectúa en lo tocante a la muerte de una persona; en este caso, en su Burguillos, cambia de registro y el poeta -el heterónimo Burguillos- escribe en seso.

Tal vez a la luz de La Dorotea y de este recorrido que hemos propuesto aquí estas afirmaciones puedan resignificarse y podamos sostener que en algunos casos la metamorfosis de la elegía pueda llegar a ser burlesca. Una vez más el texto nos demuestra ser capaz de ir un paso más allá en el reacomodamiento y en la experimentación con los sistemas genéricos. Los personajes muestran la posibilidad de una parodia o de una burla al discurso elegíaco: el lamento de Gerarda mediado por el vino, la daga de Fernando que no produce muerte sino soneto fúnebre a la tumba del retrato de Dorotea o la inserción del parlamento de un gracioso 
frente al llanto de Dorotea así lo prueban. La conjunción de las diversas materias, de los diversos géneros y de las diversas clases de personajes podrían estar mostrando, además de una teoría de la fragmentación y de la diversidad en las posibilidades de escritura, los caminos para que tanto la elegía como las demás especies poéticas provenientes de la Antigüedad clásica o del Renacimiento transitadas por Lope de Vega a lo largo de toda su obra puedan en este momento de desengaño ser tomadas bajo la forma de la parodia o de la autoparodia. Dorotea, Gerarda, Fernando y los demás personajes de La Dorotea serían así Tomés de Burguillos llevados al extremo.

\section{BiBLIOgRAFíA}

Cuenca-Godbert, Marta, 2010. "Le langage des larmes dans La Dorotea de Lope de Vega”, Bulletin hispanique, 112, $1,149-168$.

López Bueno, Begoña (coord.), 1996. La elegía, Sevilla: Universidad.

Novo, Yolanda, 1996. "La elegía en el primer tercio del s. XVII: en torno a Lope de Vega”, en: López Bueno (coord.), $1996,227-260$

Rozas, Juan Manuel, 1990. “Lope de Vega y Felipe IV en el 'ciclo de senectute”, Estudios sobre Lope de Vega, Madrid: Cátedra, 73-131.

Ruiz Pérez, Pedro, 1996. "El discurso elegíaco y la lírica barroca: pérdida y melancolía", en: López Bueno (coord.), 1996, 317-368.

Sánchez Jiménez, Antonio, 2006. "Pedro de Oña y su Arauco Domado (1596) en la obra poética de Lope de Vega", Hispanic Review, summer, 319-344.

Trueblood, Alan, 1992. "La Dorotea y la Elegía di madonna Fiammetta", en: Actas del X Congreso de la Asociación Internacional de Hispanistas, Barcelona, 21-26 de agosto de 1989. Tomo I-II / publicadas por Antonio Vilanova, Barcelona: PPU, 1139-1146.

Vega, Lope de (ed.), 2010. Elegías y elogios cortesanos (Facsímil de las ediciones príncipe h.1631-1633). Estudio y edición de Felipe Pedraza Jiménez, Madrid: Fundación Caja Castilla La Mancha, UCLM, GRISO.

Vega, Lope de (ed.), 2011. La Dorotea, Donald McGrady (ed.), Madrid: Real Academia Española.

\section{Notas}

1 No tiene sentido realizar aquí una síntesis de la gran cantidad de bibliografía existente sobre La Dorotea, para un completo estado de la cuestión se puede consultar la edición crítica del texto realizada por Donald McGrady. En su apartado acerca de la crítica del texto McGrady (2011: 425) organiza la bibliografía en nueve apartados: "su autobiografismo, su fecha de composición, sus fuentes literarias, el tiempo interno de la obra, la caracterización, el uso de refranes, sus semejanzas con otras obras de Lope, su género y su valor como literatura (lo cual incluye el papel que juega en la erudición)". Siguiendo estas divisiones para este trabajo interesa sobre todo el referido al género. Allí McGrady recorre los rótulos que ha recibido la obra desde sus primeros comentarios resaltando la "forma ambigua".

2 Felipe Pedraza, por su parte, en su edición ya citada de Elegías y Elogios cortesanos ofrece un fino estudio introductorio del Elogio en la muerte de Juan Blas de Castro y de la Elegía en la muerte de don Jerónimo de Villaisan.

3 Alan Trueblood, relaciona La Dorotea con la Elegía de Madonna Fiammeta de Boccaccio aunque con otro objetivo (pensar ambos textos como deudores de las Heroidas ovidianas) indica que la denominación de elegía transparenta "la tonalidad adolorida que presenta toda la obra”. (1992: 1140)

4 Todas las citas del texto se realizan por La Dorotea (edición 2011)

5 “Ainsi, le langage des larmes n' est pas univoque, comme un trait d' union entre la mise en scène et le cri du coeur, la rage et l' hommage tempéré, les larmes deviennent une sorte de suprême concepto.Le réseau sémiotique des larmes permet a la fois de condenser les différents étapes de l'histoire de la rupture amoureuse entre les deux protagonistes et d 'établir un parallele sans équivoque avec le désespoir de l'auteur après la mort de sa dernière maîtresse" $(2010,157)$

6 Recordemos que en el caso de las barquillas Julio no ejerce para nada el rol del gracioso sino que actúa casi como un poeta al recuperar la voz de aquel "gentilhombre al que se le ha muerto la dama" 
7 Estas construcciones de los criados además de ser patrimonio de la comedia también tienen su relación con el trasfondo celestinesco que cruza toda la obra. Así los criados, como en la Tragicomedia de Calisto y Melibea participan de las peripecias amorosas de sus amos.

8 Así lo expresa: "Le sonnet du phénix amour prend la forme d'un concepto : le tombeau littéral, poème simplement proféré, sépulcre invisible de larmes, devient monument immortel." (162) 